\title{
A novel hardware-efficient CPG model based on asynchronous cellular automaton
}

\author{
Kentaro Takeda ${ }^{1 \text { a) }}$ and Hiroyuki Torikai ${ }^{2 b)}$ \\ ${ }^{1}$ Frontier Informatics, Kyoto Sangyo University of Graduate School, \\ Motoyama, Kamigamo, Kita-ku, Kyoto 603-8555, Japan \\ ${ }^{2}$ Science and Engineering, Hosei University of Graduate School, \\ 3-7-2 Kajino-cho, Koganei, Tokyo 184-8584, Japan \\ a)takeda@mail.nsci.jp \\ b)torikai@hosei.ac.jp
}

\begin{abstract}
A novel hardware-efficient central pattern generator (CPG) model the dynamics of which is described by an asynchronous cellular automaton is proposed. It is shown that the proposed model can generate multi-phase synchronized periodic signals, which are suitable for controlling a serpentine motion of a snake-like robot. The proposed model is then implemented on a field programmable gate array (FPGA) and is used to control a snake-like robot. It is shown by experimental validation using a prototype machine that the proposed model can realize rhythmic locomotor activity in snakes. Moreover, it is shown that the proposed model consumes much fewer hardware resources (FPGA slices) than a typical conventional CPG model. Also, parameter setting methods to adjust the locomotion of the robot are shown.
\end{abstract}

Keywords: central pattern generator (CPG), field programmable gate array (FPGA), asynchronous cellular automaton

Classification: Integrated circuits

\section{References}

[1] E. Kandel, et al:: Principles of Neural Science (McGraw-Hill, 2000) 4th ed.

[2] X. Li and L. Li: "Efficient implementation of FPGA based central pattern generator using distributed arithmetic," IEICE Electron. Express 8 (2011) 1848 (DOI: 10.1587/elex.8.1848).

[3] J. Yu, et al.: "Survey on CPG-inspired control models and system implementation," IEEE Trans. Neural Netw. Learn. Syst. 25 (2014) 441 (DOI: 10.1109/TNNLS.2013.2280596).

[4] L. Righetti and A. J. Ijspeert: "Pattern generators with sensory feedback for the control of quadruped locomotion," Proc. ICRA (2008) 819 (DOI: 10.1109/ ROBOT.2008.4543306).

[5] J. H. Barron-Zambrano and C. Torres-Huitzil: "FPGA implementation of a configurable neuromorphic CPG-based locomotion controller," Neural Netw. 45 (2013) 50 (DOI: 10.1016/j.neunet.2013.04.005). 
[6] Z. Wang, et al.: "CPG-inspired locomotion control for a snake robot basing on nonlinear oscillators," J. Intell. Robot. Syst. 85 (2017) 209 (DOI: 10.1007/ s10846-016-0373-9).

[7] A. Crespi, et al.: "AmphiBot I: an amphibious snake-like robot," Robot. Auton. Syst. 50 (2005) 163 (DOI: 10.1016/j.robot.2004.09.015).

[8] T. Sato, et al.: "An adaptive decentralized control of a serpentine robot based on the discrepancy between body, brain and environment," Proc. ICRA (2010) 709 (DOI: 10.1109/ROBOT.2010.5509236).

[9] K. Takeda and H. Torikai: "A novel hardware-efficient cochlea model based on asynchronous cellular automaton dynamics: Theoretical analysis and FPGA implementation,” IEEE Trans. Circuits Syst. II, Exp. Briefs 64 (2017) 1107 (DOI: 10.1109/TCSII.2017.2672824).

[10] T. Matsubara and H. Torikai: "Asynchronous cellular automaton based neuron: Theoretical analysis and on-FPGA learning," IEEE Trans. Neural Netw. Learn. Syst. 24 (2013) 736 (DOI: 10.1109/TNNLS.2012.2230643).

[11] Y. Kuznetsov: Elements of Applied Bifurcation Theory (Springer, New York, 2004) 3rd ed.

\section{Introduction}

Central pattern generators (CPGs) are intraspinal networks of neural oscillators capable of producing rhythmic output signals to control motor systems in part of animal locomotion [1]. Many mathematical models of CPGs have been proposed and analyzed so far $[2,3,4,5,6,7,8]$. Also, CPG models have been used to control artificial robots of various kinds $[3,4,5,6,7,8]$ including snake-like robots $[5,6$, $7,8]$ like the one in Fig. 1. The purpose of this paper is to propose a novel kind of CPG model, which consumes much fewer hardware resources compared to conventional numerical integration models. First, the novel CPG model the dynamics of which is described by an asynchronous cellular automaton is proposed. By intensive numerical analyses, it is shown that the proposed model can generate multi-phase synchronized periodic signals, which are suitable for controlling a serpentine motion of a snake-like robot. The intensive numerical analyses also reveal roles of parameters. Then, the proposed model is implemented on a field programmable gate array (FPGA) and is used to control a snake-like robot. It is shown by experimental validation using a prototype machine that the proposed model can realize rhythmic locomotor activity in snakes. Moreover, it is shown that the proposed model consumes much fewer hardware resources (FPGA slices) than a typical numerical integration CPG model. Novelties and significances of this paper include the following points.

- The dynamics of most conventional CPG models are described by differential equations and are implemented as numerical integrations. On the other hand, the dynamics of the proposed CPG model is described by the asynchronous cellular automaton and is implemented as an asynchronous sequential logic circuit. Although some asynchronous cellular automaton models of biological systems have been proposed so far [9, 10], the asynchronous cellular automaton CPG model is proposed in this paper for the first time. Note that, from a fundamental research view point, such an exploration of designing a bio- 


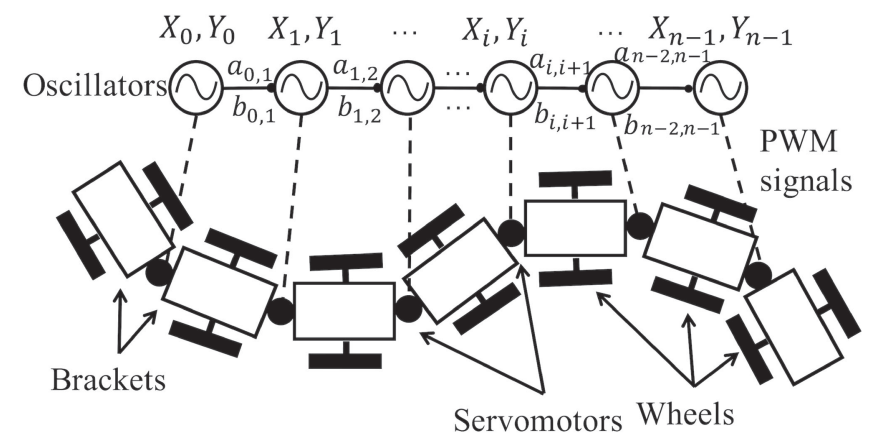

Fig. 1. Snake-like robot controlled by the proposed central pattern generator model (CPG model), which consists of asynchronous cellular automaton oscillators (ACA oscillators).

inspired engineering system (like the snake-like robot controlled by the CPG model) base on an unconventional method (like the asynchronous cellular automaton modeling method) per se is a significant research topic.

- As explained above, roles of parameters of the proposed model are revealed. From a practical view point, this is significant since these roles show how to adjust locomotion of the snake-like robot. Actually, in this paper, parameter setting methods to adjust moving velocity of the snake-like robot are shown based on the revealed roles of the parameters.

\section{Novel CPG model based on asynchronous CA}

In this section, a novel central pattern generator model (CPG model) based on an asynchronous cellular automaton is proposed. Fig. 1 shows a schematic of a snakelike robot controlled by the proposed CPG model, which consists of asynchronous cellular automaton oscillators (ACA oscillators).

\subsection{Novel asynchronous CA oscillator}

In this subsection, we propose the ACA oscillator, which has discrete states variables $X_{i} \in \mathbf{Z}_{N}=\{0, \cdots, N-1\}$ and $Y_{i} \in \mathbf{Z}_{N}$, and discrete auxiliary variables $P_{i} \in \mathbf{Z}_{M}=\{0, \cdots, M-1\}$ and $Q_{i} \in \mathbf{Z}_{M}$, where $i$ is an index for the ACA oscillators, and $N$ and $M$ are natural numbers that determine resolutions of the discrete variables $\left\{X_{i}, Y_{i}, P_{i}, Q_{i}\right\}$. In order to design a vector field of the ACA oscillator, inspired by [7], the following functions $g_{i}: \mathbf{Z}_{N} \times \mathbf{Z}_{N} \rightarrow \mathbf{R}$ and $h_{i}: \mathbf{Z}_{N} \times$ $\mathbf{Z}_{N} \rightarrow \mathbf{R}$ are prepared: $g_{i}\left(X_{i}, Y_{i}\right)=-\omega_{i}\left(\left(X_{i}-\frac{N}{2}\right)^{2}+\left(Y_{i}-\frac{N}{2}\right)^{2}-\delta_{i}\right)\left(X_{i}-\frac{N}{2}\right) \delta_{i}^{-1}-$ $\omega_{i}\left(Y_{i}-\frac{N}{2}\right)$ and $h_{i}\left(X_{i}, Y_{i}\right)=-\omega_{i}\left(X_{i}-\frac{N}{2}\right)$, where $\delta_{i} \in \mathbf{R}$ and $\omega_{i} \in \mathbf{R}$ are parameters. Note that the functions $g_{i}$ and $h_{i}$ are not implemented as hardware but are used to design the following discrete functions $G_{i}: \mathbf{Z}_{N} \times \mathbf{Z}_{N} \rightarrow \mathbf{Z}_{M}^{ \pm}=\{-(M-1), \cdots$, $0, \cdots, M-1\}$ and $H_{i}: \mathbf{Z}_{N} \times \mathbf{Z}_{N} \rightarrow \mathbf{Z}_{M}^{ \pm}$.

$$
G_{i}\left(X_{i}, Y_{i}\right)=\left\lfloor\frac{\alpha_{x}}{g_{i}\left(X_{i}, Y_{i}\right)}\right\rfloor, \quad H_{i}\left(X_{i}, Y_{i}\right)=\left\lfloor\frac{\alpha_{y}}{h_{i}\left(X_{i}, Y_{i}\right)}\right\rfloor,
$$

where the functions $G_{i}$ and $H_{i}$ are assumed to be saturated at $\pm(M-1)$ hereafter, i.e., $G_{i}\left(X_{i}, Y_{i}\right)=(M-1)$ for $(M-1) \leq\left\lfloor\frac{\alpha_{x}}{g_{i}\left(X_{i}, Y_{i}\right)}\right\rfloor, \quad G_{i}\left(X_{i}, Y_{i}\right)=-(M-1)$ for $-(M-1) \geq\left\lfloor\frac{\alpha_{x}}{g_{i}\left(X_{i}, Y_{i}\right)}\right\rfloor, H_{i}\left(X_{i}, Y_{i}\right)=(M-1)$ for $(M-1) \leq\left\lfloor\frac{\alpha_{y}}{h_{i}\left(X_{i}, Y_{i}\right)}\right\rfloor, H_{i}\left(X_{i}, Y_{i}\right)=$ $-(M-1)$ for $-(M-1) \geq\left\lfloor\frac{\alpha_{y}}{h_{i}\left(X_{i}, Y_{i}\right)}\right\rfloor$, and $\lfloor$.$\rfloor denotes the floor function. The ACA$ 


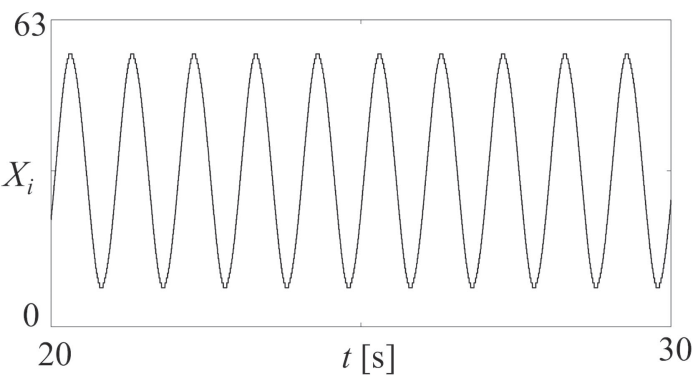

(a)

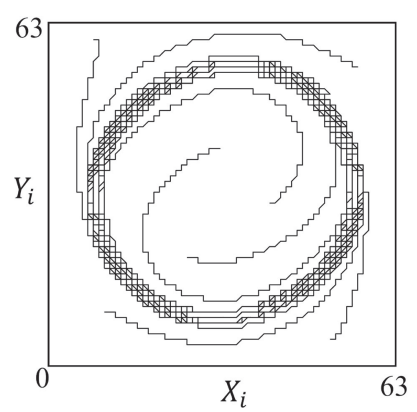

(b)

Fig. 2. (a) Typical time waveform of the proposed asynchronous cellular automaton oscillator. $\left(N, M, T_{X}, T_{Y}, \alpha_{x}, \alpha_{y}, \delta_{i}, \omega_{i}\right)=$ $\left(2^{6}, 2^{10}, 0.0001,0.00015 \sqrt{1.00020134}, 10000, \frac{100000}{15 \sqrt{1.00020134}}\right.$, $\left.25^{2}, 2 \pi \times 1.0\right)$. (b) Phase plane trajectories for different initial states. The mean radius is $r_{i} \simeq 25$.

oscillator has two internal clocks $C_{X}(t)$ and $C_{Y}(t)$, which are described by $C_{X}(t)=1$ if $t=0, T_{X}, 2 T_{X} \ldots$ and $C_{X}(t)=0$ otherwise; and $C_{Y}(t)=1$ if $t=$ $0, T_{Y}, 2 T_{Y} \ldots$ and $C_{Y}(t)=0$ otherwise, where $T_{X} \in \mathbf{R}^{+}=\{t \mid t \geq 0\}$ and $T_{Y} \in \mathbf{R}^{+}$ are periods of $C_{X}(t)$ and $C_{Y}(t)$, respectively. In this paper, the clock generators are assumed to be uncoupled and thus the clocks $C_{X}(t)$ and $C_{Y}(t)$ are assumed to be asynchronous. These asynchronous internal clocks $C_{X}(t)$ and $C_{Y}(t)$ trigger the following asynchronous transitions of the discrete auxiliary variables $P_{i}$ and $Q_{i}$, respectively.

$$
\begin{aligned}
& \text { If } C_{X}(t)=1, \text { then } P_{i}\left(t_{+}\right):= \begin{cases}P_{i}(t)+1 & \text { if } P_{i}(t)<\left|G_{i}\left(X_{i}(t), Y_{i}(t)\right)\right|, \\
0 & \text { if } P_{i}(t) \geq\left|G_{i}\left(X_{i}(t), Y_{i}(t)\right)\right|,\end{cases} \\
& \text { If } C_{Y}(t)=1, \text { then } Q_{i}\left(t_{+}\right):= \begin{cases}Q_{i}(t)+1 & \text { if } Q_{i}(t)<\left|H_{i}\left(X_{i}(t), Y_{i}(t)\right)\right|, \\
0 & \text { if } Q_{i}(t) \geq\left|H_{i}\left(X_{i}(t), Y_{i}(t)\right)\right|,\end{cases}
\end{aligned}
$$

where the symbol " $t_{+}$" denotes " $\lim _{\epsilon \rightarrow+0} t+\epsilon$ " and the symbol " $=$ " denotes an "instantaneous state transition" hereafter. In addition, the asynchronous internal clocks $C_{X}(t)$ and $C_{Y}(t)$ trigger the following asynchronous transitions of the discrete state variables $X_{i}$ and $Y_{i}$, respectively.

$$
\begin{aligned}
& \text { If } C_{X}(t)=1 \text { and } P_{i}(t) \geq\left|G_{i}\left(X_{i}(t), Y_{i}(t)\right)\right| \text {, then } \\
& X_{i}\left(t_{+}\right):= \begin{cases}X_{i}(t)+1 & \text { if } X_{i}(t) \neq N-1 \text { and } G_{i}\left(X_{i}(t), Y_{i}(t)\right) \geq 0, \\
X_{i}(t)-1 & \text { if } X_{i}(t) \neq 0 \text { and } G_{i}\left(X_{i}(t), Y_{i}(t)\right)<0,\end{cases} \\
& \text { If } C_{Y}(t)=1 \text { and } Q_{i}(t) \geq\left|H_{i}\left(X_{i}(t), Y_{i}(t)\right)\right| \text {, then } \\
& Y_{i}\left(t_{+}\right):= \begin{cases}Y_{i}(t)+1 & \text { if } Y_{i}(t) \neq N-1 \text { and } H_{i}\left(X_{i}(t), Y_{i}(t)\right) \geq 0, \\
Y_{i}(t)-1 & \text { if } Y_{i}(t) \neq 0 \text { and } H_{i}\left(X_{i}(t), Y_{i}(t)\right)<0 .\end{cases}
\end{aligned}
$$

Fig. 2(a) shows a typical time waveform of the discrete state variable $X_{i}$ and Fig. 2(b) shows phase plane trajectories for different initial states. It can be seen that the ACA oscillator oscillates in a steady state. In order to characterize such an oscillation, let us introduce the following definitions (see also Fig. 3(a)).

Definition 1a: Assume the discrete state variable $X_{i}$ is in a steady state. Assume the discrete state variable $X_{i}$ ever repeats the following two behaviors: (i) $X_{i}$ increases and transits into a certain constant value $X_{C}$ and (ii) $X_{i}$ decreases and 


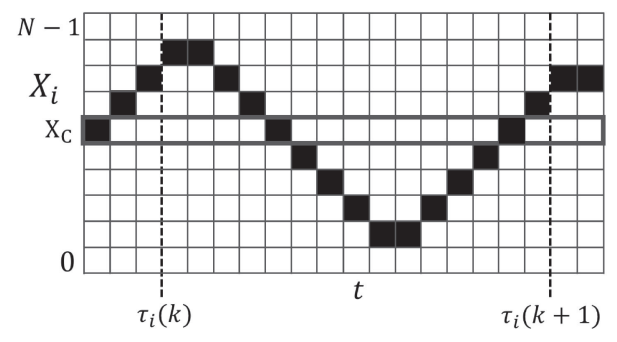

(a)

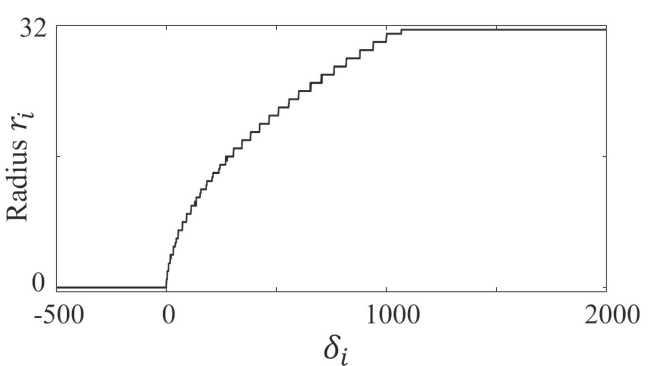

(b)

Fig. 3. (a) Definition of $\tau_{i}(k)$, which is the moment when $X_{i}$ transits into a local maximum value. (b) Characteristics of the mean radius $r_{i}$ of the discrete state variable $X_{i}$ with respect to the parameter $\delta_{i} . \quad\left(N, M, T_{X}, T_{Y}, \alpha_{x}, \alpha_{y}, \omega_{i}\right)=\left(2^{6}, 2^{10}, 0.0001\right.$, $\left.0.00015 \sqrt{1.00020134}, 10000, \frac{100000}{15 \sqrt{1.00020134}}, 2 \pi \times 1.0\right)$.

transits from $X_{C}$. Under these assumptions, let $\tau_{i}(k)$ denote the moment when the discrete state variable $X_{i}$ transits to its maximum value from the moment when (i) occurs to the moment when the next (i) occurs. Then, the discrete state variable $X_{i}$ is said to have a mean radius $r_{i}$ defined by

$$
r_{i}=\frac{1}{2 K} \sum_{k=1}^{K} \max _{\tau_{i}(k) \leq t<\tau_{i}(k+1)}\left\{X_{i}(t)\right\}-\min _{\tau_{i}(k) \leq t<\tau_{i}(k+1)}\left\{X_{i}(t)\right\},
$$

where $K$ is a sufficiently large positive integer.

Definition 1b: Assume the discrete state variable $X_{i}$ is in a steady state and ever stays in a certain constant value $X_{C}$. Then, the discrete state variable $X_{i}$ is said to have a mean radius $r_{i}=0$.

For example, the discrete state variable $X_{i}$ in Fig. 2 has a mean radius $r_{i} \simeq 25.0$. Fig. 3(b) shows the characteristics of the mean radius $r_{i}$ with respect to the parameter $\delta_{i}$. It can be seen that the ACA oscillator does not oscillate for $\delta_{i}<0$ and oscillates for $\delta_{i}>0$. Hence the ACA oscillator exhibits a bifurcation near $\delta_{i}=0$. Since the mean radius $r_{i}$ is approximately proportional to $\sqrt{\delta_{i}}$ for $\delta_{i}>0$ near $\delta_{i}=0$, the bifurcation is similar to the supercritical Hopf-bifurcation [11]. Since our goal is to design the CPG model, the ACA oscillator is expected to oscillate and thus the parameter $\delta_{i}$ should be set to a positive value. Then we assume the assumptions in Definition 1a. Under these assumptions, let us give the following definition.

Definition 2: Assume the assumptions in Definition 1a are satisfied. Then, the discrete state variable $X_{i}$ is said to have a mean frequency $f_{i}$ defined by

$$
f_{i}=\frac{K}{\sum_{k=1}^{K} \tau_{i}(k+1)-\tau_{i}(k)} .
$$

For example, the discrete state variable $X_{i}$ in Fig. 2 has a mean frequency $f_{i} \simeq 1.0$. Fig. 4 shows the characteristics of the mean frequency $f_{i}$ with respect to the parameter $\omega_{i}$ and Table I shows the mean frequency $f_{i}$ for different values of the period $T_{Y}$ of the internal clock $C_{Y}(t)$. Based on these characteristics, roles of the parameters can be summarized as follows. 


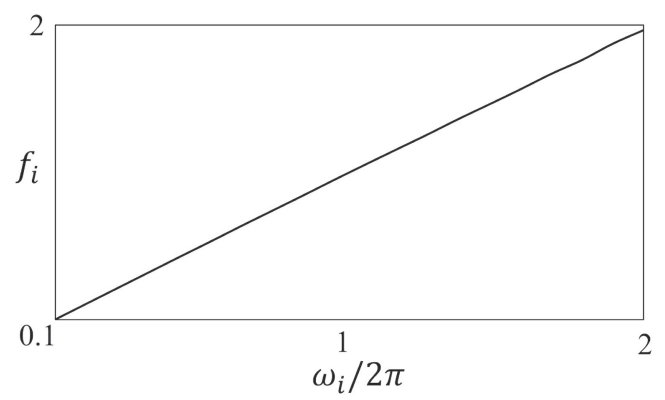

Fig. 4. Characteristics of the mean frequency $f_{i}$ of the discrete state variable $X_{i}$ with respect to the parameter $\omega_{i}$. $\left(N, M, T_{X}\right.$, $\left.T_{Y}, \alpha_{x}, \alpha_{y}, \delta_{i}\right)=\left(2^{6}, 2^{10}, 0.0001,0.00015 \sqrt{1.00020134}, 10000\right.$, $\left.\frac{100000}{15 \sqrt{1.00020134}}, 25^{2}\right)$.

Table I. Periods $\left(T_{X}, T_{Y}\right)$ of the clocks and the mean frequency $f_{i}$ of the discrete state variable $X_{i} .\left(N, M, \delta_{i}, \omega_{i}\right)=\left(2^{6}, 2^{10}, 25^{2}\right.$, $2 \pi \times 1.0)$.

\begin{tabular}{ccc|c}
\hline Internal clocks & Period $T_{X}$ & Period $T_{Y}$ & Mean frequency $f_{i}$ \\
\hline Frequency locked & 0.0001 & 0.00015 & 1.00004800520048031 \\
Asynchronous & 0.0001 & $0.00015 \sqrt{1.00020134}$ & 1.00000000000000000 \\
\hline
\end{tabular}

- The mean radius $r_{i}$ of the discrete state variable $X_{i}$ can be adjusted by the parameter $\delta_{i}$ as shown in Fig. 3(b).

- The mean frequency $f_{i}$ of the discrete state variable $X_{i}$ can be adjusted by the parameter $\omega_{i}$ as shown in Fig. 4.

- The mean frequency $f_{i}$ of the discrete state variable $X_{i}$ can be fine-tuned by the parameter $T_{Y}$ as shown in Table I.

\subsection{Novel central pattern generator model}

In this subsection, the novel CPG model, which consists of coupled $n$ ACA oscillators, is proposed. In order to couple the ACA oscillators, the discrete function $G_{i}$ in Eq. (1) is modified as follows.

$$
G_{i}\left(X_{0}, \cdots, X_{n-1}, Y_{0}, \cdots, Y_{n-1}\right)=\left\lfloor\frac{\alpha_{x}}{g_{i}\left(X_{i}, Y_{i}\right)+\sum_{j=0}^{n-1}\left(a_{i, j} Y_{j}+b_{i, j} X_{j}\right)}\right\rfloor,
$$

where $a_{i, j}$ and $b_{i, j}$ are coupling constants between the $i$-th and the $j$-th ACA oscillators, respectively, and $i, j \in\{0,1, \cdots, n-1\}$. In this paper, the following coupling constants are focused on.

$$
a_{i, i+1}=k_{a} \text { and } b_{i, i+1}=k_{b} \text { for } i=0,1, \cdots, n-2 \text {, }
$$

where other $a_{i, j}$ and $b_{i, j}$ are zeros. For example, the CPG model in Fig. 1 is characterized by such coupling constants. Fig. 5 shows typical time waveforms of the proposed CPG model. It can be seen that the ACA oscillators oscillate with the same mean frequency in Fig. 5(a) and with different mean frequencies in Fig. 5(b). In order to characterize such oscillations, let us introduce the following definition. 


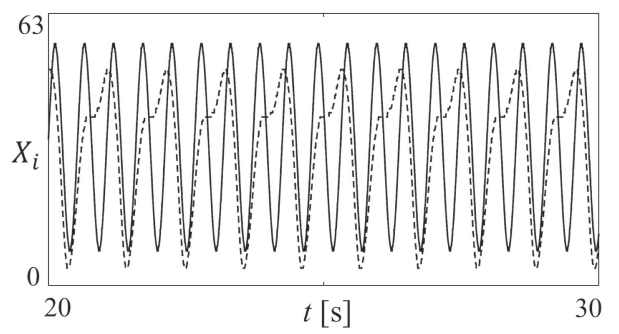

(a)

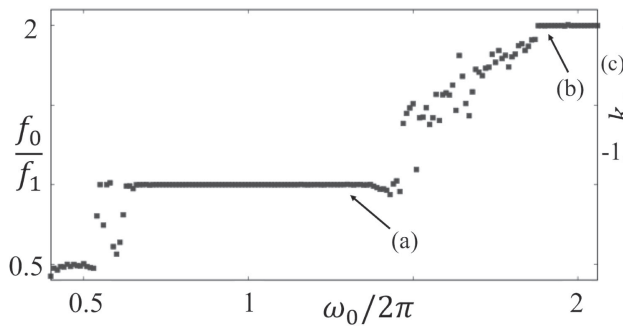

(c)

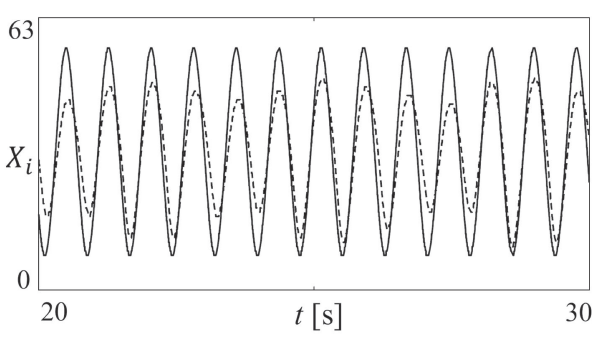

(b)

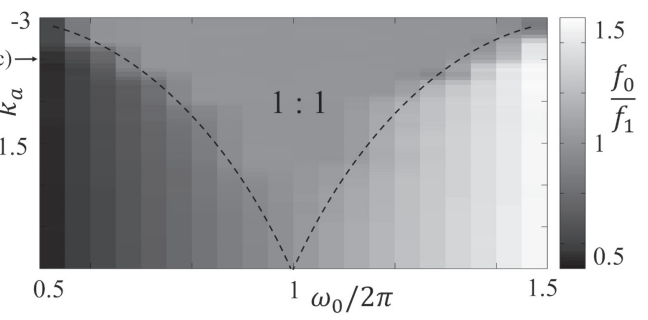

(d)

Fig. 5. Typical time waveforms of the CPG model with $n=2$ ACA oscillators and their characteristics. $\left(N, M, T_{X}, T_{Y}, \alpha_{x}, \alpha_{y}\right.$, $\left.\delta_{0}, \delta_{1}, \omega_{1}\right)=\left(2^{6}, 2^{10}, 0.0001,0.00015 \sqrt{1.00020134}, 10000\right.$, $\left.\frac{100000}{15 \sqrt{1.00020134}}, 25^{2}, 25^{2}, 2 \pi \times 1.0\right) . k_{b}=0$. In (a) and (b), the solid curves and the dashed curves show discrete states variables $X_{0}$ and $X_{1}$, respectively. (a) $1: 1$ synchronization. $\left(\omega_{0}, k_{a}\right)=$ $(2 \pi \times 1.3,-2.5)$. (b) $2: 1$ synchronization. $\left(\omega_{0}, k_{a}\right)=(2 \pi \times$ $1.9,-2.5)$. (c) Characteristic of the mean frequency ratio $\frac{f_{0}}{f_{1}}$ with respect to the parameter $\omega_{0} . k_{a}=1.0$. The arrows (a) and (b) correspond to the figures (a) and (b), respectively. (d) Characteristic of the mean frequency ratio $\frac{f_{0}}{f_{1}}$ with respect to the parameters $\left(\omega_{0}, k_{a}\right)$. The arrow (c) corresponds to the figure (c).

Definition 3: If the ratio $f_{i}: f_{j}$ of the mean frequencies $f_{i}$ and $f_{j}$ of the $i$-th and the $j$-th ACA oscillators can be represented by a ratio $n: m$ of integers $n$ and $m$, then the ACA oscillators are said to exhibit an $n: m$ synchronization. In this case, $\frac{f_{i}}{f_{j}}$ is referred to as a mean frequency ratio of the $i$-th and the $j$-th ACA oscillators.

For example, the ACA oscillators in Fig. 5(a) exhibit an 1: 1 synchronization with the mean frequency ratio $\frac{f_{0}}{f_{1}}=1$ and the ACA oscillators in Fig. 5(b) exhibit a $2: 1$ synchronization with the mean frequency ratio $\frac{f_{0}}{f_{1}}=2$. Fig. 5(c) shows the characteristics of the mean frequency ratio $\frac{f_{0}}{f_{1}}$ with respect to the parameter $\omega_{0}$. Fig. 5(d) shows the characteristics of the mean frequency ratio $\frac{f_{0}}{f_{1}}$ with respect to the parameters $\left(\omega_{0}, k_{a}\right)$. In this figure, the parameter region labeled by $1: 1$ leads to the $1: 1$ synchronization. Note that in this paper we design the CPG model, which utilizes the $1: 1$ synchronization. So, the $1: 1$ synchronization is focused on hereafter. Fig. 6 shows various 1:1 synchronizations with different phase differences. In order to characterize such phenomena, let us introduce the following definition (see also Fig. 7).

Definition 4: Assume the assumptions in Definition 1a are satisfied and assume the ACA oscillators in the CPG model exhibit $1: 1$ synchronizations. Then

$$
\phi_{i, j}=\frac{1}{K} \sum_{k=1}^{K}\left(\left(\tau_{i}(k)\left(\bmod f_{i}^{-1}\right)\right)-\left(\tau_{j}(k)\left(\bmod f_{i}^{-1}\right)\right)\right)
$$




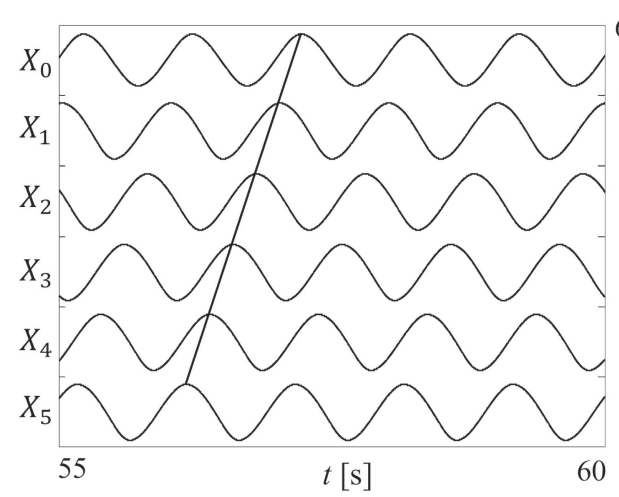

(a)

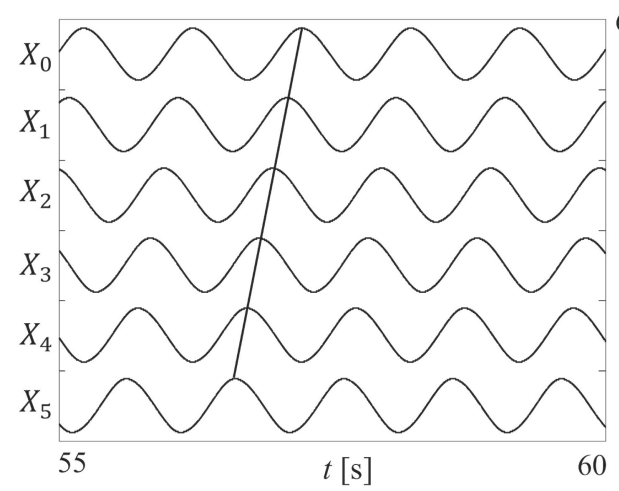

(c)

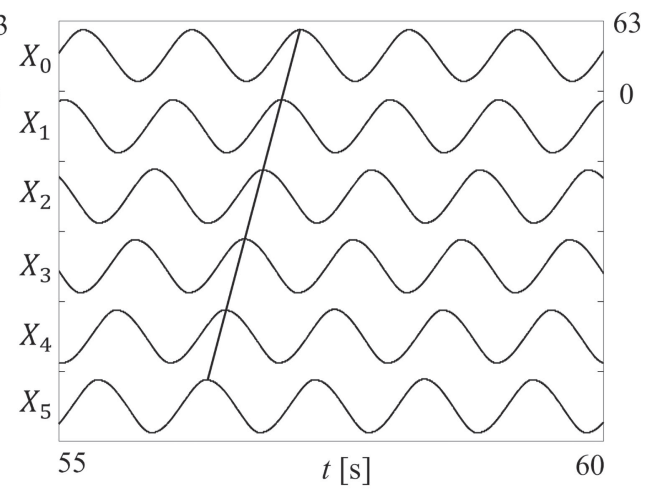

(b)

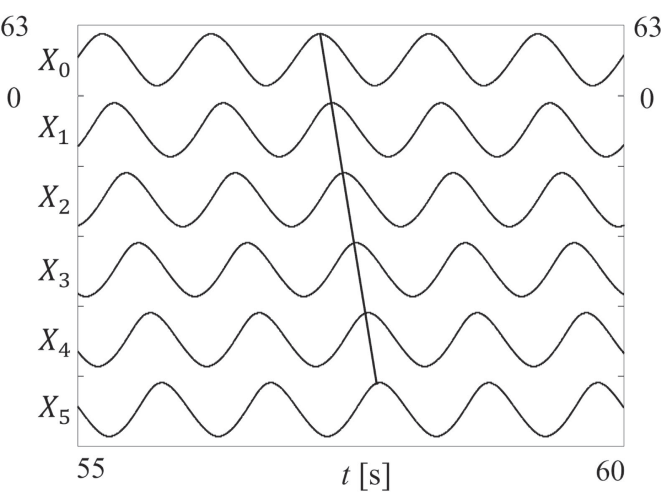

(d)

Fig. 6. $1: 1$ synchronizations of the proposed $\mathrm{CPG}$ with various mean phase differences $\Phi . \quad\left(N, M, T_{X}, T_{Y}, \alpha_{x}, \alpha_{y}, \delta_{i}, \omega_{i}\right)=\left(2^{6}, 2^{10}\right.$, $0.0001,0.00015 \sqrt{1.00020134}, 10000, \frac{100000}{15 \sqrt{1.00020134}}, 25^{2}, 2 \pi \times$ 1.0) for all $i$. $\left(n, k_{b}\right)=(6,0.3)$. (a) $k_{a}=-1.0$. $\Phi=1.071$. (b) $k_{a}=-0.5 . \Phi=0.849$. (c) $k_{a}=-0.3 . \Phi=0.599$. (d) $k_{a}=0.3$. $\Phi=-0.472$.

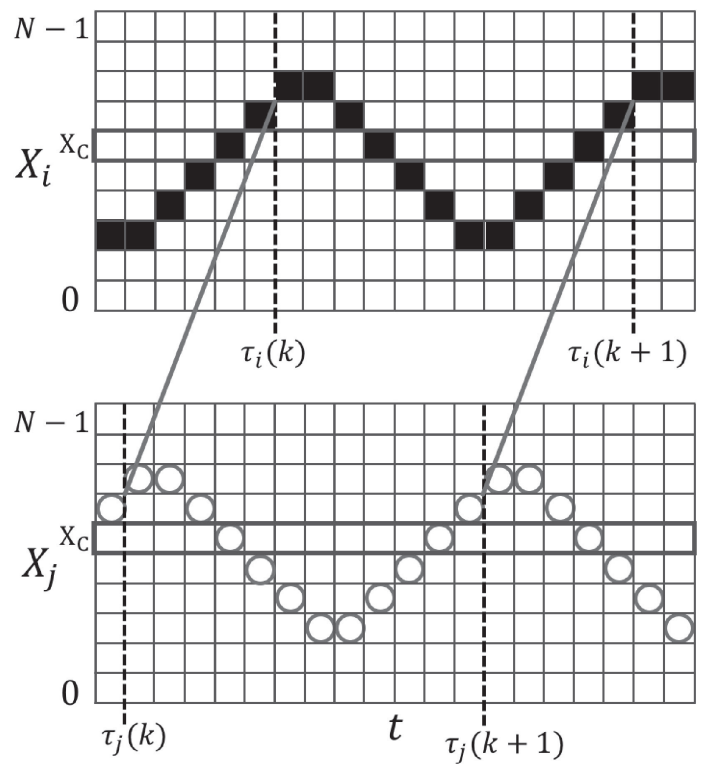

Fig. 7. Definition of the phase difference $\phi_{i, j}$. The arrows represent $\tau_{i}(k)-\tau_{j}(k)$ and $\tau_{i}(k+1)-\tau_{j}(k+1)$. 


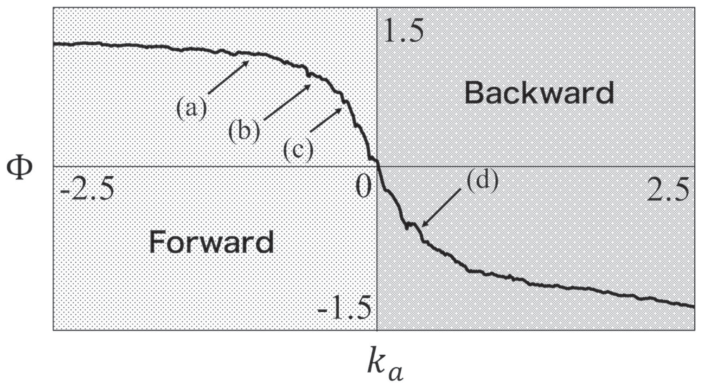

Fig. 8. Characteristics of the mean phase difference $\Phi$ with respect to the parameter $k_{a} . \quad\left(N, M, T_{X}, T_{Y}, \alpha_{x}, \alpha_{y}, \delta_{i}, \omega_{i}\right)=\left(2^{6}, 2^{10}\right.$, $0.0001,0.00015 \sqrt{1.00020134}, 10000, \frac{100000}{15 \sqrt{1.00020134}}, 25^{2}, 2 \pi \times$ $1.0)$ for all $i . \quad\left(n, k_{b}\right)=(6,0.3)$. The parameter regions "Forward" and "Backward" lead to forward and backward moving directions of the snake-like robot in Fig. 9. The arrows (a), (b), (c), and (d) correspond to Figs. 6(a), (b), (c), and (d), respectively.

is referred to as a phase difference between the $i$-th and the $j$-th ACA oscillators. Also,

$$
\Phi=\frac{1}{n-1} \sum_{i=0}^{n-2} \phi_{i, i+1}
$$

is referred to as a mean phase difference of the ACA oscillators in the CPG model.

For example, the ACA oscillators in Figs. 6(a), (b), (c) and (d) have mean phase differences $\Phi=1.071,0.849,0.599$ and -0.472 , respectively. Fig. 8 shows the characteristics of the mean phase difference $\Phi$ with respect to the parameter $k_{a}$. Based on the characteristics of the CPG model in Figs. 5 and 8 and the characteristics of the ACA oscillator in Fig. 3(b), Fig. 4, and Table I, roles of the parameters from a viewpoint of the CPG model for controlling the snake-like robot in Fig. 1 can be summarized as follows.

- The amplitude of the oscillating rotary motion of the servomotor can be adjusted by the parameter $\delta_{i}$.

- The frequency of the oscillating rotary motion of the servomotor can be adjusted by the parameter $\omega_{i}$ and fine-tuned by the parameter $T_{Y}$.

- The phase difference of the oscillating rotary motions of the servomotors can be adjusted by the parameter $k_{a}$. Since the moving direction (forward or backward) of the snake-like robot is determined by the phase difference (see Fig. 8), it can be adjusted by the parameter $k_{a}$.

- The moving velocity of the snake-like robot can be adjusted by the frequency and the phase differences of the oscillating rotary motions of the servomotors under the condition that the amplitudes of the oscillating rotary motions are appropriately set. Therefore, the moving velocity of the snake-like robot can be adjusted by the parameters $\left(\omega_{i}, k_{a}\right)$ under an appropriate setting of the value of the parameter $\delta_{i}$.

Hence, in the next section, effects of the parameters $\left(\omega, k_{a}\right)$ to the moving velocity of the snake-like robot are analyzed. 


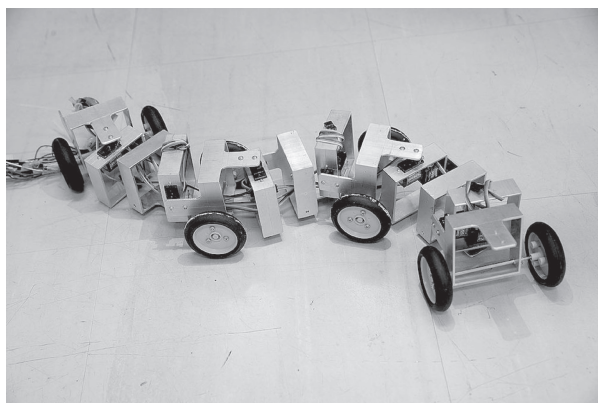

(a)

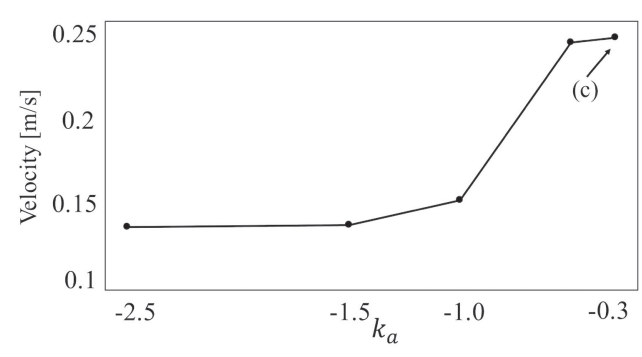

(b)

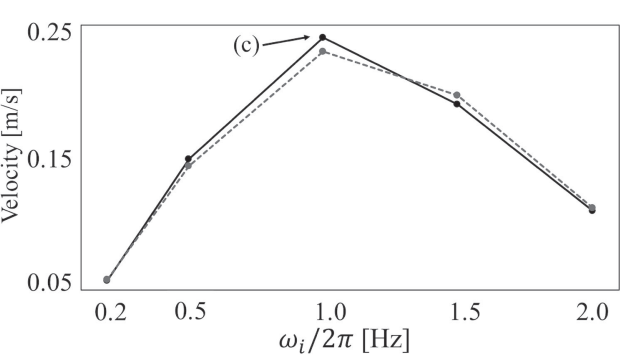

(c)

Fig. 9. Snake-like robot controlled by the proposed CPG model. $\left(N, M, T_{X}, T_{Y}, \alpha_{x}, \alpha_{y}, \delta_{i}\right)=\left(2^{6}, 2^{10}, 0.0001,0.00015,10000\right.$, $\left.6667,25^{2}\right)$ for all $i$. $\left(n, k_{b}\right)=(6,0.3)$. (a) Picture of the implemented snake-like robot. (b) The solid graph shows the characteristics of the moving velocity of the snake-like robot with respect to the parameter $k_{a} . \omega_{i}=2 \pi \times 1$ for all $i$. (c) The solid graph shows the characteristics of the moving velocity of the snake-like robot with respect to the parameter $\omega_{i} . k_{a}=$ -0.3 . In (b) and (c), the dashed graphs show the characteristics of the moving velocity of the snake-like robot controlled by a differential equation CPG model [7].

\section{Hardware implementation and comparison}

Recall that the dynamics of the proposed CPG model is described by Eqs. (2)-(5). These equations are written in a VHDL code, which is compiled into a bitstream file by using Xilinx's design software environment Vivado 2016. 2. The resulting bitstream file is downloaded to Xilinx's field programmable gate array (FPGA) XC7K325T-2FFG900C. Fig. 9(a) shows a picture of the snake-like robot controlled by the FPGA-implemented CPG model. As shown in the figure, the snake-like robot consists of $n=6$ sections and an extra bracket-B attached to the head of the snake, and each section consists of two brackets, bracket-A and bracket-B. Each $i$-th bracket-A has Tower Pro's servo motor SG92R, the target angle of which is instructed by the discrete state variable $X_{i}$ of the CPG via a pulse width modulation. Figs. 9(b) and (c) show experimentally measured characteristics of the moving velocity of the robot with respect to the parameters $k_{a}$ and $\omega_{i}$, respectively. From these figures, it can be concluded that the moving velocity of the snake-like robot can be controlled by adjusting the parameters $k_{a}$ and $\omega_{i}$.

For comparison, a CPG model consisting of a simple oscillator [7], the bifurcation mechanism of which is similar to the ACA oscillator, is implemented by the same compiler and the same FPGA device. The oscillator is described by a 
Table II. Comparison. The symbol "\#" denotes "the number of."

\begin{tabular}{c|cc}
\hline $\begin{array}{c}\text { Hardware resources } \\
\text { occupied in the FPGA }\end{array}$ & Proposed CPG model & $\begin{array}{c}\text { CPG model consisting of } \\
\text { the ODE oscillator [7] }\end{array}$ \\
\hline \# Look-up-tables & 12824 & 48216 \\
\# Flip-flops & 1035 & 816 \\
\# Total slices & 3633 & 13519 \\
\hline
\end{tabular}

set of two ordinary differential equations (ODEs), which is implemented as a forward Euler formula in a VHDL code. The bit-length of the ODE oscillator is decreased as short as possible under the condition that the resulting characteristics of the moving velocity of the snake-like robot are almost identical with those in Figs. 9(b) and (c) (see the dashed graphs in these figures). Table II shows comparison of hardware resources used to implement the CPG models in the FPGA device. It can be seen that the proposed CPG model consumes much fewer hardware resources compared to the CPG model consisting of the ODE oscillator.

\section{Conclusions}

The novel CPG model based on the asynchronous cellular automaton was proposed. The proposed CPG model was implemented on the FPGA and was used to control the snake-like robot. By experimental validation using the prototype machine, it was shown that the proposed model can realize rhythmic locomotor activity in snakes. Moreover, it was shown that the proposed CPG model and the Hopf-oscillator CPG model (which is one of simple CPG models) realize almost identical characteristics of the moving speeds of the snake-like robot but the proposed model consumes much fewer hardware resources. Also, the parameter setting methods to adjust the moving speeds of the snake-like robot were clarified. Future problems are including (a) comparison to other models, (b) more detailed analysis on the hardware cost, and (c) applications to other types robots.

\section{Acknowledgment}

This work was supported by KAKENHI Grant Numbers 15K00352 and 18K11482. 